\title{
Knowledge Management for Complex Product Development
}

\author{
Framework and Implementation
}

\author{
Alexander Smirnov ${ }^{1}$, Alexey Kashevnik ${ }^{1}$, Nikolay Teslya ${ }^{1}$, Nikolay Shilov ${ }^{1}$, \\ Andreas Oroszi ${ }^{2}$, Mario Sinko ${ }^{2}$, Michael Humpf ${ }^{2}$, and Jens Arneving ${ }^{2}$ \\ ${ }^{1}$ St.Petersburg Institute for Informatics and Automaton of the Russian Academy of Sciences \\ (SPIIRAS) \\ 39, 14 line, 199178, St.Petersburg, Russia \\ \{smir, alexey, teslya, nick\}@iias.spb.su \\ ${ }^{2}$ Festo AG \& Co. KG, \\ Plieninger Straße 50, Ostfildern-Scharnhausen, 73760, Germany \\ \{oro, sni, hump, arv\}@de. festo.com
}

\begin{abstract}
Complex product development process in global companies usually consists of several stages involving different software systems and numerous users from various departments and centers. Automation of this process assumes changes in company's workflows, data models and software systems used. As a result, implementation of such changes in large companies faces a lot of difficulties because business process cannot be stopped to switch between old and new workflows, old and new software systems, etc. The paper represents an ongoing long-term project aimed at knowledge management for multi-level complex product development at Festo AG\&Co KG. It is based on the central product ontology describing product classification, their properties and configuration \& compatibility restrictions. The developed system complex significantly simplifies and speeds up the process of product development and maintenance.
\end{abstract}

Keywords: product knowledge management, product design, complex product configuration.

\section{Introduction}

Current trends in the worldwide economy and increasing competition require companies to propose not only new products but whole integrated solutions to their customers. Such solutions might consist of multiple physical devices as well as services. One of the consequences of this is appearance of "complex products", which consist of other products (both regular products and complex products) and often include software units using different services.

Another strategy that brings companies and their customers in a closer collaboration is innovation democratisation. This is a relatively new term standing for involvement of customers into the process of designing and creating new products 
and services. This makes it possible for companies to better meet needs of their customers. (Hippel, 2006)

Modularisation is one of the keys to carry out mass customisation and innovation democratization strategies with low cost (Egan, 2004; Sharda and Voß, 2005). Module is a result of disassembling functions and architecture of the product. It can provide the end-products in wider scope while decreasing diversification in product components.

In this situation the previously developed PLM mechanisms and workflows supporting products from the design phase to putting them to the market and further has appeared to be inefficient. As a result, there was a need to design new, knowledge-based workflows and supporting software systems to increase efficiency of designing and maintaining new product ranges. Knowledge-based PLM is currently a critical and strategic issue (Bernard and Tichkievitch, 2008) and the company recognizes this (e.g., Smirnov and Shilov, 2011).

However, implementation of such changes in large companies faces many difficulties because business process cannot be stopped to switch between old and new workflows, old and new software systems have to be supported at the same time, the range of products, which are already in the markets, has to be maintained in parallel with new products, etc. Another problem is that it is difficult to estimate in advance which solutions and workflow would be efficient and convenient for the employees. Hence, just following existing PLM implementation guidelines is not possible (also confirmed by Bokinge and Malmqvist, 2012), and this process has to be and iterative and interactive.

The paper represents an ongoing long-term project aimed at knowledge management for multi-level complex product development at Festo AG\&Co KG company that has more than 300000 customers in 176 countries supported by more than 52 companies worldwide with more than 250 branch offices and authorized agencies in further 36 countries.

Fig. 1 represents the developed multi-level knowledge management concept for complex product development, which is the core of the described project. The black rectangles have been already implemented and the appropriate tools are indicated (grey areas).

The major product ontology is in the center of the model. It is used to solve the problem of knowledge heterogeneity and enables interoperability between heterogeneous information sources due to provision of their common semantics and terminology (Uschold and Grüninger, 1996). It describes all the products (produced and to be produced) and their features (existing and possible). This ontology is supported by two tools addressed in sec. 2: NOC (New Order Code) and CONCode ("old code", CON is a prefix based on the reverse acronym of New Order Code). Building a knowledge map that connects this ontology to different knowledge sources of the company is yet to be addressed. 


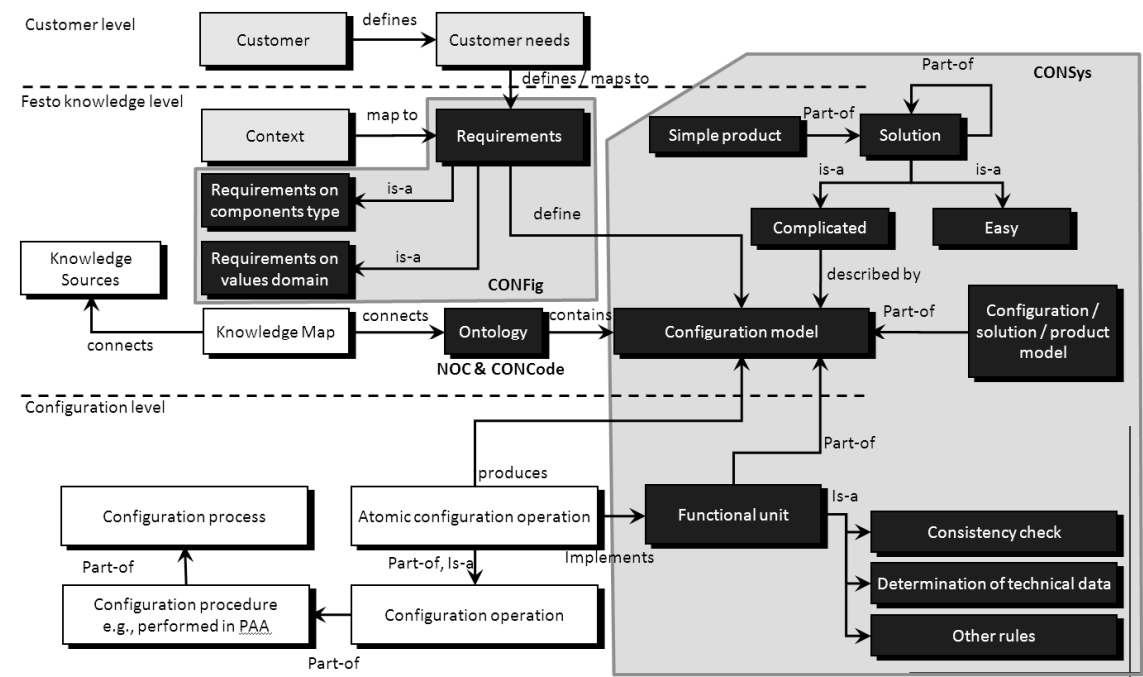

Fig. 1. Multi-level knowledge management for complex product development

The configuration level has also been addressed only partially so far. The tool called CONSys (SYStems of products) supports creation of configuration models of complex products and solutions (sec. 3). Configuration models include their structures as well as different rules responsible for complex product consistency checks, technical data calculation, etc. The support of the configuration process design (the left lower part of the figure) has not been implemented yet.

Based on the built configuration model the process of complex product or solution configuration in accordance with given requirements can be automated. This is done via the tool called CONFig (CONFIGuration) addressed in sec. 4. So far this tool supports the configuration process in terms used within the company (Festo knowledge level). In reality, the customers are used to operate different terminology (Customer level), which doesn't correspond "one to one" to that used within the company. Besides, customers from different industries can also operate different terms. As a result, there is a need to create configuration tools that can map customers' requirements to those used in the company taking into account the context (customer's industry segment, history of customer's orders, etc.). This is the goal of the future research.

\section{Product Ontology}

The developed approach is based on the idea that knowledge can be represented by two levels. The first level describes the structure of knowledge. Knowledge represented by the second level is an instantiation of the first level knowledge; this knowledge holds object instances.

The knowledge of the first level (structural knowledge) is described by a common ontology of the company's product families (classes). Ontologies provide a common way of knowledge representation for its further processing. They have shown their usability for this type of tasks (e.g., Bradfield et al., 2007; Chan and Yu, 2007; Patil et al., 2005) 
The first step to implementation of the approach is creation of the ontology. This operation was done automatically based on existing documents and defined rules of the model building. The resulting ontology consists of more than 1000 classes organized into a 4 level taxonomy, which is based on the VDMA classification (Verband Deutscher Maschinen- und Anlagenbau, German Engineering Federation, VDMA, 2013). Taxonomical relationships support inheritance that makes it possible to define more common attributes for higher level classes and inherit them for lower level subclasses. The same taxonomy is used in the company's PDM and ERP systems.

For each product family (class) a set of properties (attributes) is defined, and for each property its possible values and their codes are defined as well. The lexicon of properties is ontology-wide, and as a result the values can be reused for different families. Application of the common single ontology provides for the consistency of the product codes and makes it possible to reflect incorporated changes in the codes instantly.

The ontology is multilingual. Each class and attribute can be assigned names in different languages so that specialists from different countries could work simultaneously preserving consistency of the ontology.

The selectable properties can be communicated to the customers with so called configuration solutions or product selectors. These tools can be used in electronic catalogues and online shop systems. As a result, a new product codification scheme based on product families and characteristics has been developed (Oroszi et al., 2009). For companies with wide assortments of products (more than $30000-40000$ products of approx. 700 types, with various configuration possibilities) it is very important to ensure that customers can easily navigate among them. This is an important task for customer communication management because well defined and understandable product identification is mandatory for ensuring good corporate look for the company (Baumeister, 2002; Fjermestad and Romano, 2002; Piller and Schaller, 2002). The system should be organised in such a way that would ensure the uniqueness of the code and its validity. The resulting code is supplied into the company's PDM and ERP system.

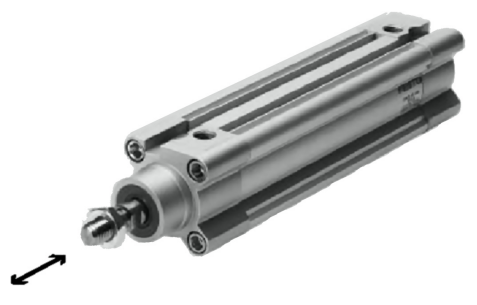

Fig. 2. DSBC product: a pneumatic drive

The described above ontology provides rules for the codification system in the following way. For each class a number of attributes is assigned in a certain sequence. This sequence of attributes forms a template for codes of products belonging to the appropriate product family. For each product the properties are replaced with codes of their values corresponding to the particular product to generate its code.

For example, the DSBC series is a family of pneumatic drives with a single moving rod (Fig. 2). There are 35 different properties with each between 4 to 10 values. Customer and engineers can select from these properties and values. Some combinations of different properties and values are not allowed because they are technically impossible. 
Typical properties of the DSBC series are "locking in end positions" and "special antifriction features". Their possible values are presented in Table 1.

Given code template consisting of a delimiter and the above two properties, the following codes can be built:

- Standard (no locking, no antifriction features): DSBC

- "Extend / retract" (locking in both directions) and no antifriction features: DSBC-E1

- "Extend / retract" and "Low friction": DSBC-E1L

Fig. 3 represents a fragment of the real code scheme for the DSBC series. Inheritance of more common properties from higher, more abstract classes ensures that for different branches of the classification the sequence of common properties will be the same that simplifies the code interpretation.

After finding the desired product based on its properties, the set of selected properties is translated into a product code based on the existing code scheme of its class (product type). After transmitting the product code to the company, the customer receives an order confirmation listing selected product properties. The confirmation is generated with the company's ERP system, which uses code schemes to translate product codes into the properties of product.

Table 1. Compatibility table

\begin{tabular}{lll}
\hline Value Code & Value Name & Description \\
\hline \multicolumn{2}{l}{ Locking in end positions } \\
& Without (Standard) & the most common choice, no locking in end positions \\
E1 & Extend / Retract & locking is applied in both directions of movement \\
E2 & Extend & locking is applied in the direction of extension \\
E3 & Retract & locking is applied in the direction of retraction \\
Special antifriction features & \\
& None / Normal type & the most common choice, no special antifriction features \\
L & Low friction & product with reduced friction \\
S & Slow speed & product oriented to slow movement
\end{tabular}

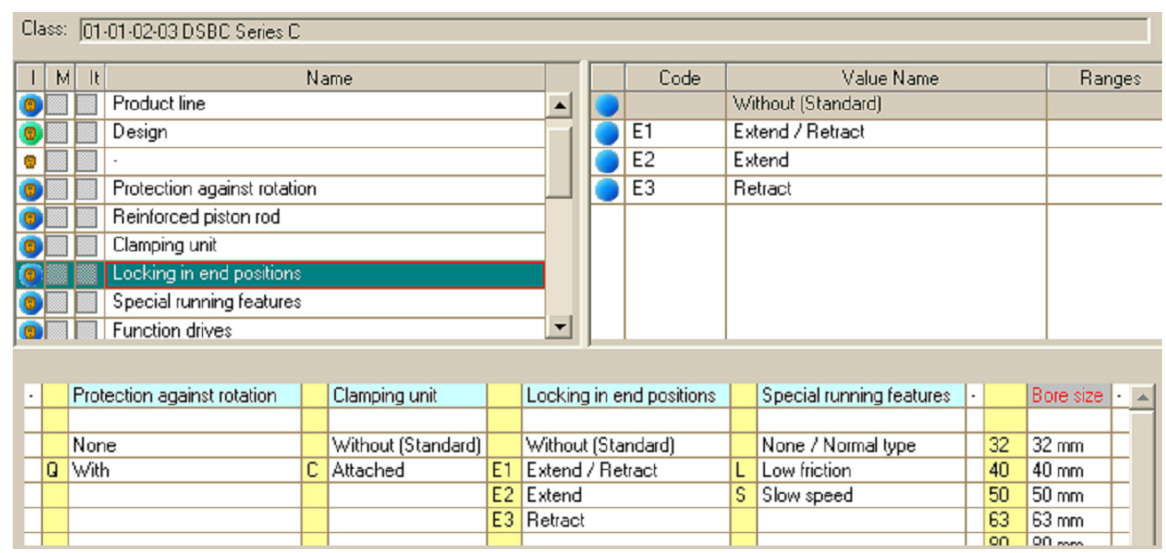

Fig. 3. Code scheme for the DSBC series (a fragment) 


\section{Complex Product Configuration Rules}

As it was already mentioned, today, the customers tend to buy complete customized solutions (referred to as "complex products") consisting of numerous products, rather than separate products. Before, the complex products were configured by experts based on the customer requirements. Today, this process requires automation. However, inter-product relationships are very challenging. For example, the most common use case is the relationship between a main product and an accessory product. While both products are derived from different complex products there are dependencies which assign a correct accessory to a configured main product. The dependencies are related to the products' individual properties and values. E.g., "1 $1 \times 3 / 2$ or $2 \times 3 / 2$-way valve" cannot be installed on a valve terminal if its size is "Size 10, deviating flow rate 1". The depth of productaccessory relationships is not limited, so accessory-of-accessory combinations have also to be taken into account. The relationships can be very complex when it comes to define the actual location and orientation of interfaces and mounting points between products.

Complex product description consists of two major parts: product components and rules. Complex product components can be the following: simple products, other complex products, and application data. The set of characteristics of the complex product is a union of characteristics of its components. The rules of the complex products are union of the rules of its components plus extra rules. Application data is an auxiliary component, which is used for introduction of some additional characteristics and requirements to the product (for example, operating temperatures, certification, electrical connection, etc.). They affect availability and compatibility of certain components and features via defined rules.

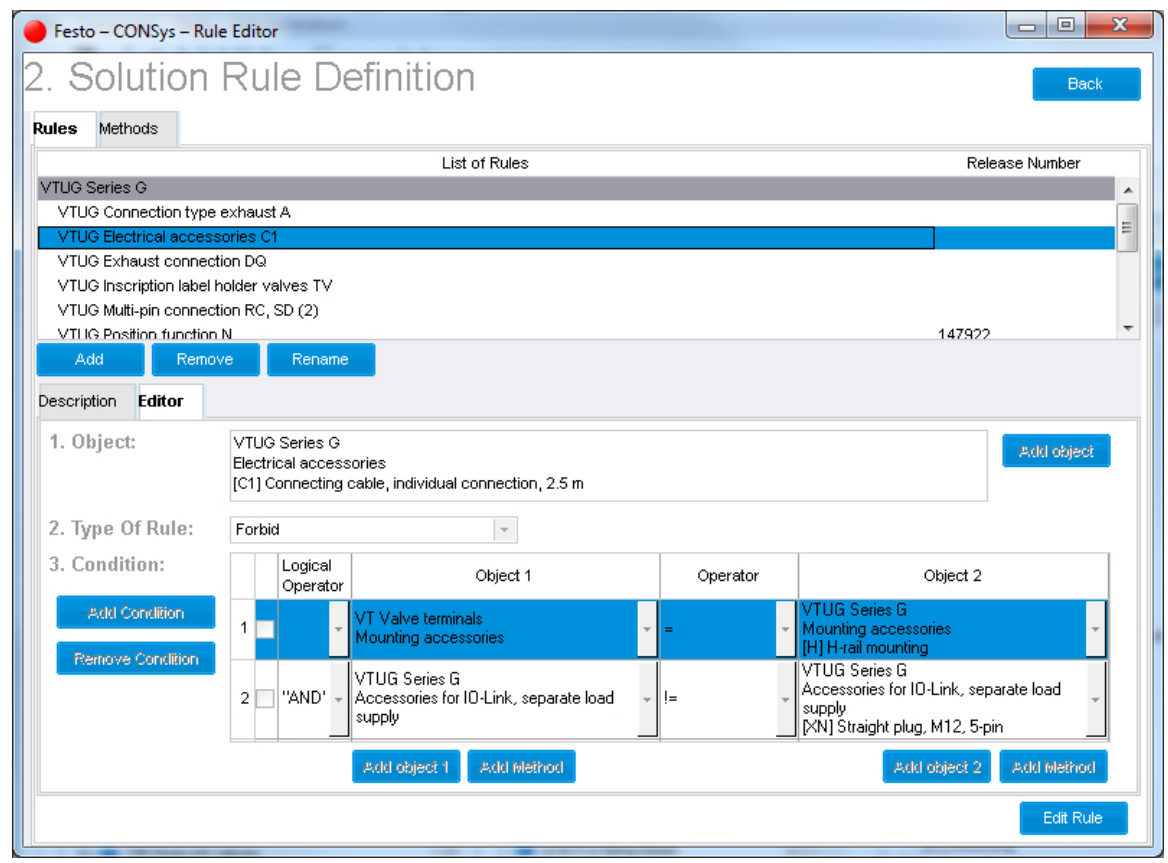

Fig. 4. Solution rules example 
Some example rules are shown in Fig. 4. The figure represents a valve terminal (VTUG) and compatibility of electrical accessories option C1 (individual connecting cable) with mounting accessories (compatible only with H-rail mounting) and accessories for input-output link (not compatible with 5 pin straight plug M12). These rules are stored in the database and can be later used during configuration of the valve terminal for certain requirements.

\section{Product Configuration}

When the configuration model is finished it is proposed to the customers so that they could configure required products and solutions themselves or with assistance of product managers.

An example configuration interface is presented in Fig. 5. The figure represents the mentioned above valve terminal VTUG with an electrical control module CTEU. The user can choose values (from drop down lists, outlined in Fig. 5 with bold dotted line) for different properties (rows in the table). Depending on the choices other properties either selected automatically, disabled or become hidden. This is done on the basis of the rules described during the complex product definition. The rules are processed "on the fly", i.e. as soon as the user chooses a value, all other values are checked for consistency immediately.

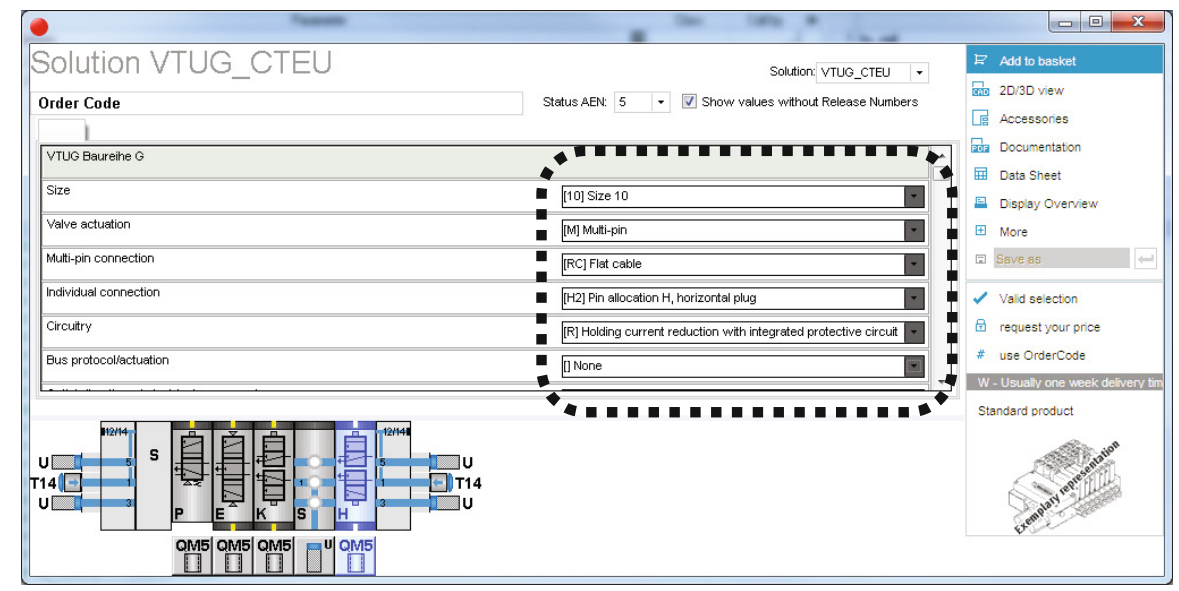

Fig. 5. Product configuration interface: an example

\section{$5 \quad$ Integrated Workflow}

So far developed integrated knowledge management workflow is presented in Fig. 6. At the first stage the major product ontology is filled with generic classifications of products and their components. This is done via two tools (NOC and CONCode) since recently developed order code scheme differs from that used before. However, 
since multiple customers are used to operate with the old classification it has to be maintained.

At the next stage the product managers and modelers design new products and solutions based on existing products and components (the CONSys tool). If a new product or component is needed, its implementation can be requested from the order code structure team. Together with new products and solutions, the appropriate rules and conditions are designed as well (e.g., acceptable load, size, compatibility constraints, etc.).

When the configuration model is finished it is proposed to the customers so that they could configure required products and solutions themselves or with assistance of product managers (the CONFig tool).

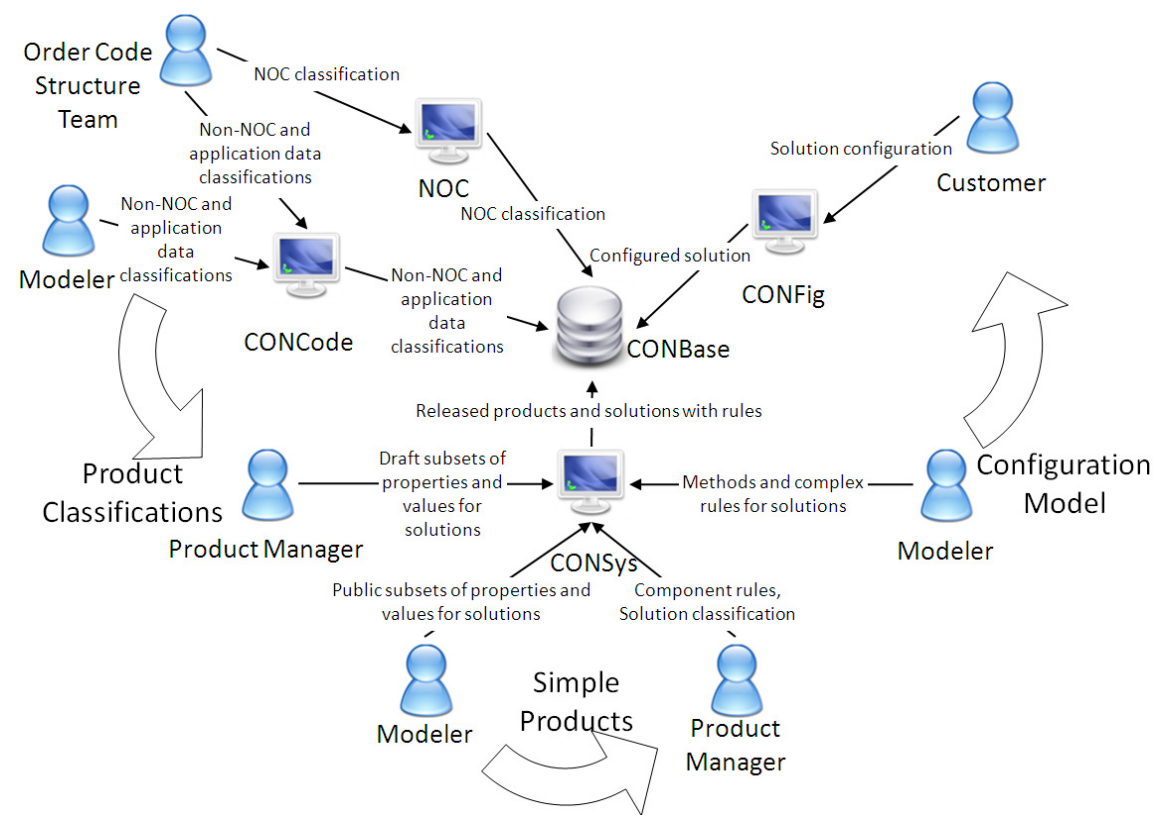

Fig. 6. Integrated workflow

\section{Conclusion}

The paper presents a long-term ongoing project aimed at introduction of product knowledge management for different stages of its lifecycle. The systems significantly simplify and speed up the process of product development. The technical options presented by the product manager and developer are converted into order-relevant options. As most of the characteristics can be used again, only new options must be discussed and entered in the system. Besides this, the error risk has been significantly reduced. 
The major advantages of the developed systems are: systematic order codes for all products; machine readability; quick orientation for selecting right products and services; security when selecting and ordering products and services.

One other advantage is the reusability of the data. The structured data are used in other processes such as:

- Automatically creating master data in SAP models;

- Automatically creating data for the configuration models and services;

- Automatically generating an ordering sheet for the print documentation (this ordering sheet was generated earlier with high expenditure manually);

- Automatically generating a product and service list which is needed in the complete process implementing new products.

Acknowledgments. The research presented is motivated by a joint project between SPIIRAS and Festo AG\&Co KG. Some parts of the work have been sponsored by grants \# 12-07-00298-a, \# 12-07-00302-a of the Russian Foundation for Basic Research, project \# 213 of the research program "Intelligent information technologies, mathematical modelling, system analysis and automation" of the Russian Academy of Sciences, and project 2.2 "Methodology development for building group information and recommendation systems" of the basic research program "Intelligent information technologies, system analysis and automation" of the Nanotechnology and Information technology Department of the Russian Academy of Sciences.

\section{References}

Baumeister, H.: Customer relationship management for SMEs. In: Proceedings of the 2nd Annual Conference eBusiness and eWork e2002, Prague, Czech Republic (2002)

Bokinge, M., Malmqvist, J.: PLM implementation guidelines - relevance and application in practice: a discussion of findings from a retrospective case study. International Journal of Product Lifecycle Management 6(1), 79-98 (2012)

Bernard, A., Tichkievich, S. (eds.): Methods and Tools for Effective Knowledge Life-CycleManagement, 588.p. Springer (2008)

Bradfield, D.J., Gao, J.X., Soltan, H.: A Metaknowledge Approach to Facilitate Knowledge Sharing in the Global Product Development Process. Computer-Aided Design \& Applications 4(1-4), 519-528 (2007)

Chan, E.C.K., Yu, K.M.: A framework of ontology-enabled product knowledge management. International Journal of Product Development 4(3/4), 241-254 (2007)

Egan, M.: Implementing a Successful Modular Design. In: Proceedings of the 7th Workshop on Product Structuring - Product Platform Development, pp. 49-58 (2004)

Fjermestad, J., Romano Jr., N.C.: An Integrative Implementation Framework for Electronic Customer Relationship Management: Revisiting the General Principles of Usability and

Resistance. In: Proceedings of the 36th Hawaii International Conference on System Sciences (HICSS 2003), Big Island, HI, USA (2003) ISBN 0-7695-1874-5 - Track 7

Von Hippel, E.: Democratizing innovation, p. 208. The MIT Press, Cambridge (2006) 
Oroszi, A., Jung, T., Smirnov, A., Shilov, N., Kashevnik, A.: Ontology-Driven Codification for Discrete and Modular Products. Int. J. of Product Development 8(2), 162-177 (2009)

Patil, L., Dutta, D., Sriram, R.: Ontology-based exchange of product data semantics. IEEE Transactions on Automation Science and Engineering 2(3), 213-225 (2005) ISSN: 15455955

Piller, F., Schaller, C.: Individualization Based Collaborative Customer Relationship Management: Motives, Structures, and Modes of Collaboration for Mass Customization and CRM. Working Paper No. 29 of the Dept. of General and Industrial Management, Technische Universität München ISSN 0942-5098, http://www. masscustomization.de/

download/ / TUM-AIBWP029.pdf (May 2002, 2004)

Sharda, R., Voß, S.: Information and Management Systems for Product Customization. Integrated Series in Information Systems, 270. p. Springer (2005); ( ISBN 978-0-38723347-5 (Print) 978-0-387-23348-2 (Online)

Smirnov, A., Shilov, N.: Collaborative Recommendation Systems for PLM: Approach and Technological Framework. In: Proc. of the 8th International Product Lifecycle Management Conference, PLM 2011, Eindhoven, The Netherlands (2011)

Uschold, M., Grüninger, M.: Ontologies: Principles, methods and applications. Knowledge Engineering Review 11(2), 93-155 (1996)

VDMA, German Engineering Federation (2013), http : / /www . vdma . org / en_GB / 